\title{
Obtención de Dímeros por Termólisis de 1,2-Propanodiol
}

\author{
Nathaly Milanés, Adolfo E. Ensuncho y Juana Robles \\ Universidad de Córdoba, Departamento de Química, Grupo de Química Computacional, \\ Cra 6 № 74-103, Córdoba-Colombia (e-mail: namios20@gmail.com; adem9abril@hotmail.com, \\ juanarobles2003@yahoo.com)
}

Recibido Nov. 02, 2011; Aceptado Ene. 12, 2012; Versión final recibida May. 25, 2012

\section{Resumen}

Los compuestos dímeros 3-oxi-(bis)-2-propanol, 2-(2-hidroxipropoxi)-propanol y 2-oxi-(bis)propanol fueron obtenidos de la reacción de descomposición térmica del 1,2-propanodiol, realizada a $573.15 \mathrm{~K}$ y a su presión de vapor, utilizando un sistema de reacción a microescala, Los dímeros resultaron ser isómeros y fueron elucidados por cromatografía gaseosa acoplada a espectrometría de masas. Estos compuestos no han sido reportados en la literatura como productos de termólisis de polioles. La cinética y el mecanismo, fueron confirmados por cálculos computacionales utilizando el método de la Teoría del Funcional Densidad, DFT. El mecanismo propuesto transcurrió a través de un proceso concertado de una sola etapa, que pasa por un estado de transición cíclico de cuatro miembros, compatible con cálculos cinéticos y termodinámicos.

Palabras clave: mecanismo de reacción, termólisis, dimerización, espectrometría de masas, 1,2propanodiol

\section{Synthesis of Dimers from Thermolysis of 1,2-Propanediol}

\begin{abstract}
The dimers 3-oxy-(bys)-2-propanol, 2-(2-hydroxypropoxy)-propanol and 2-oxy-(bys)-propanol were obtained from the thermal decomposition reaction of 1,2-propanediol, at $573.15 \mathrm{~K}$ and to its vapor pressure, and using microscale reaction system. The dimers resulted to be isomers and were elucidated by gas chromatography coupled to mass spectrometry. These compounds have not been reported in literature as products polyol thermolysis. The kinetic and the mechanism were validated by computational calculations using the Density Functional Theory, DFT. The proposed mechanism occurred in a single step concerted process that passes through a four member transition cyclical state that is compatible with kinetic and thermodynamic calculations.
\end{abstract}

Keywords: reaction mechanism, thermolysis, dimerization, mass spectrometry, 1,2-propanediol 


\section{INTRODUCCIÓN}

El compuesto 1,2-propanodiol es un líquido claro, incoloro, viscoso, que tiene baja volatilidad y alto punto de ebullición, en su forma pura es muy higroscópico (fácilmente recoge y emite agua); por eso es usado en la preparación de mezclas agua/alcoholes alifáticos del tipo diol, los cuales son utilizados como cosolventes en la industria farmacéutica para incrementar la solubilidad en solución acuosa de fármacos con baja solubilidad en agua (Rytting et al., 2005; Kawakami et al., 2006; Buggins et al., 2007). También es empleado como materia prima en la industria de los polímeros por ser precursor de algunas fibras sintéticas utilizadas en la industria textil (Rogulska et al., 2007). Los polioles son utilizados en la industria biomédica como aditivos en la síntesis de biopolímeros (Pawlowski y Rokicki, 2004; Rokicki y Piotrowska, 2002). Dada las aplicaciones, usos e importancia de los dioles, se han realizado estudios teóricos y experimentales, donde se analiza desde un punto de vista estructural y termodinámico, las interacciones de estos dioles en solución acuosa y en fase gas. Dentro de estos trabajos, se realizó un estudio teórico de las estructuras quirales del 1,2-polioles en monómeros, dímeros e hidratos, observandose la importancia del enlace de hidrógeno para estabilizar los conformeros en cada sistema (Friedemann, 1995). Se estudió la estructura del 1,2-etanodiol por difracción de rayos $\mathrm{X}$ y cálculos teóricos, mostrando que la estructura cristalina no posee enlaces de hidrógeno intermolecular, exhibiendo una conformación gauche estable que presenta una hiperconjugación (Deepak, 2010). Del mismo modo, se estudió por dinámica molecular en fase gas y acuosa, la formación de clústeres, donde se mostró el papel que juega el enlace de hidrógeno y la función que cumple la molécula de agua en el proceso de asociación en la trayectoria de formación de los clúster del 1,2-octanodiol, 1,2-etanodiol y 1,2-hexanodiol (Friedemann, 1997). Asimismo, se estudiaron los efectos de las interacciones de los grupos funcionales en las reacciones bimolécular y disociación de algunos dioles, en donde la distribución relativa de los productos formados de la reacción entre una molécula del diol y el ión dimetil éter, depende de la posición de los grupo $\mathrm{OH}$. En los dioles con los grupos hidroxilicos cercanos forman un producto cíclico de sustitución y para los compuestos con los grupos hidroxilicos separados los productos formados son de disociación (Eichmann, 1992). Aunque hay mayor número de reportes en la literatura, sobre la descripción de las interacciones inter e intramolecular de estos dioles, los estudios cinéticos han recibido menor atención (Ye, 2012). Así, un estudio reciente reporta la descomposición unimolécular del etilenglicol por cálculos teóricos (ab initio) con altos niveles de refinamiento por el método $\mathrm{QCISD}(\mathrm{T}) / \mathrm{CBS} / / \mathrm{B} 3 \mathrm{LYP} / 6-31 \mathrm{G}++\mathrm{G}(\mathrm{d}, \mathrm{p})$, donde se muestran resultados de la cinética y descomposición del etilenglicol, los cuales predicen la importancia de la eliminación de agua porque favorece la formación de moléculas como aldehídos y enoles. Asimismo, las constantes de velocidad calculadas indican que las reacciones de eliminación de agua predominan a bajas temperaturas, mientras que en altas se presenta disociación con ruptura de enlace carbono-carbono (Ye, 2012). De igual forma, estudios experimentales de la pirolisis de algunos alcoholes polihidroxílicos, se realizaron utilizando un sistema estático de alto vacío y presentaron un mecanismo de reacción complejo (Chuchani et al., 1997). Del mismo modo, se estudió la cinética de termólisis del 2,5-hexanodiol, mostrando como producto formado el compuesto 2,5-dimetilfurano. La descomposición térmica resulto ser unimolecular, homogénea y de primer orden, con constante de velocidad $(k)$ del orden de $10^{-4} \mathrm{~s}^{-1}$ (Ensuncho, et al., 2011). Sin embargo, a pesar de la importancia de estos dioles a nivel industrial, biomédico y estudios experimentales y teóricos realizados de estos compuestos, aún faltan muchos aspectos por investigar. Con este trabajo, se pretende contribuir en el estudio de las características y propiedades de las reacciones de termólisis del compuesto 1,2-propanodiol. En razón a lo anterior, se realizó la termólisis del 1,2-propanodiol a la temperatura de 573.15K. Ya que ellos pertenecen a la familia de los alcoholes; los cuales han despertado gran interés como alternativa energética por su poder de combustión y estos compuestos también podrían ser una buena alternativa energética.

\section{METODOLOGÍA}

\section{Sección experimental}

El 1,2-propanodiol (1,2-PDO) (producto comercial Aldrich), fue secado con $\mathrm{Na}_{2} \mathrm{SO}_{4}$, decantado y destilado bajo presión reducida obteniéndose una pureza del $99 \%$ la cual se verificó por 
cromatografía gaseosa acoplada a espectrometría de masas (GC-EM) en un equipo Thermo Electrón DSQII Single Quadrupole GC/MS, utilizando una columna de 30m de longitud, con diámetro interno de $0.32 \mathrm{~mm}$, espesor de película de $0.25 \mu \mathrm{m}$ y limite de temperatura de $598.15 \mathrm{~K}$ específica para alcoholes. Con respecto a la termólisis del compuesto 1,2-PDO se llevó a cabo en un minireactor químico provisto de un horno cilíndrico recubierto de asbesto y material refractario acoplado a una termocupla cuya temperatura es controlada electrónicamente (Lafont, 2009, Ensuncho, et al., 2011). El horno además posee 18 perforaciones con diámetro de $4 \mathrm{~mm}$ cada una, sobre las cuales se introdujeron ampollas conteniendo una alícuota de $25 \mu \mathrm{L}$ de 1,2-PDO. Estas muestras fueron sometidas a una temperatura de $573.15 \mathrm{~K}$ por un tiempo de 10 días, ya que se realizaron pruebas a temperaturas y tiempos menores pero no se observó la aparición de señales significativas correspondientes a productos. Por otro lado, a temperaturas mayores de $573.15 \mathrm{~K}$, los capilares no soportaron la presión ejercida por el reactivo.

\section{Detalles Computacionales}

Los cálculos se llevaron a cabo usando la versión para Linux del paquete de programas Gaussian 03 (Frisch, 2004). Las geometrías de reactivos, estados de transición (ETs) y productos designados como $\mathrm{P}_{1-1}, \mathrm{P}_{1-2}$, y $\mathrm{P}_{2-2}$, respectivamente, fueron completamente optimizadas con el funcional híbrido de tres parámetros de Becke y el funcional de correlación con gradiente corregido de Lee, Yang y Parr (B3LYP) (Janik et al., 2006; Balci y Akyuz, 2008; llieva et al., 2010), usando el conjunto base estándar $6-31+G(d, p)$ (Csonka, 2002), con funciones de polarización para los átomos pesados y funciones difusas para los átomos ligeros. Las estructuras de los ETs designados como $\mathrm{ET}_{1-1^{\prime}}, \mathrm{ET}_{1-2}$ y $\mathrm{ET}_{2-2}$, respectivamente, se localizaron usando el método QST2 (Rincón y Toro-Labbé, 2007), el cual emplea la aproximación de tránsito sincrónico cuadrático para acercarse a la región cuadrática del estado de transición y posteriormente a través del algoritmo cuasi-Newton finaliza la optimización. Además, se calcularon frecuencias vibracionales para verificar la naturaleza de los puntos estacionarios localizados sobre la superficie de energía potencial B3LYP, como mínimos o estados de transición de primer orden al presentar solo una frecuencia imaginaria. Asimismo para cada estado de transición se realizaron cálculos de "Coordenada Intrínseca de Reacción" (CIR) para verificar que dichos estados de transición localizados conectaban con los correspondientes puntos mínimos estacionarios asociados a reactantes y productos (Contreras y Gerli, 2008; Mohajeri y Jenabi, 2007). En cuanto al análisis termoquímico de los diferentes mecanismos propuestos como es usual, las entalpías y energías libres de Gibbs de activación se calcularon teniendo en cuenta las sumas y diferencias apropiadas en cada caso (Ochterski, 2000). El paquete Gaussian 03 proporciona las sumas de la energía electrónica y la corrección térmica a la entalpia, así como la corrección térmica a la energía libre de Gibbs, de modo que, simplemente tomando la diferencia de estos valores para reactivos y estados de transición se pueden obtener dichos parámetros termodinámicos. Así por ejemplo, mediante las ecuaciones 1 y 2, la entalpía y energía libre de Gibbs de activación pueden ser calculadas a la temperatura de trabajo (573.15K).

$$
\begin{aligned}
& \Delta H^{\ddagger}(573.15 K)=\left(\varepsilon_{0}+H_{\text {corr }}\right)_{E T}-\sum\left(\varepsilon_{0}+H_{\text {corr }}\right)_{\text {Reacivios }} \\
& \Delta G^{\ddagger}(573.15 K)=\left(\varepsilon_{0}+G_{\text {corr }}\right)_{E T}-\sum\left(\varepsilon_{0}+G_{\text {corr }}\right)_{\text {Reacivios }}
\end{aligned}
$$

Asimismo las constantes de velocidad pueden ser calculadas a través de la ecuación 3 asumiendo que el coeficiente de transmisión usado en la teoría clásica del estado de transición corresponde a uno (Quijano et al., 2006; Janik et al., 2006).

$k(T)=\frac{k_{B}{ }^{T}}{h c^{\circ}} e^{-\frac{\Delta G^{\ddagger}}{R T}}$

Donde $\mathrm{k}_{\mathrm{B}}$ es la constante de Boltzmann, $T$ es la temperatura absoluta, $\mathrm{h}$ la constante de Planck, $\mathrm{R}$ la constante universal de los gases, $\Delta G^{\ddagger}$ la energía libre de activación para la descomposición 
térmica de 1,2-PDO y c ${ }^{\circ}$ la concentración molar estándar que puede ser tomada como 1. En todos los casos fue usado el factor de conversión; 1 Hartree $=627.510 \mathrm{kcal} \mathrm{mol}^{-1}$ (Levine et al., 2001).

\section{RESULTADOS Y DISCUSIÓN}

La termólisis del 1,2-propanodiol presentó una reacción de dimerización en la cual se obtuvieron tres productos principales nombrados como 3-oxi-(bis)-2-propanol, 2-(2-hidroxipropoxi)-propanol y 2-oxi-(bis)-propanol y designados como $P_{1-1}, P_{1-2}$ y $P_{2-2^{\prime}}$. Estos productos fueron analizados y elucidados por cromatografía de gases acoplada a espectrometría de masas.

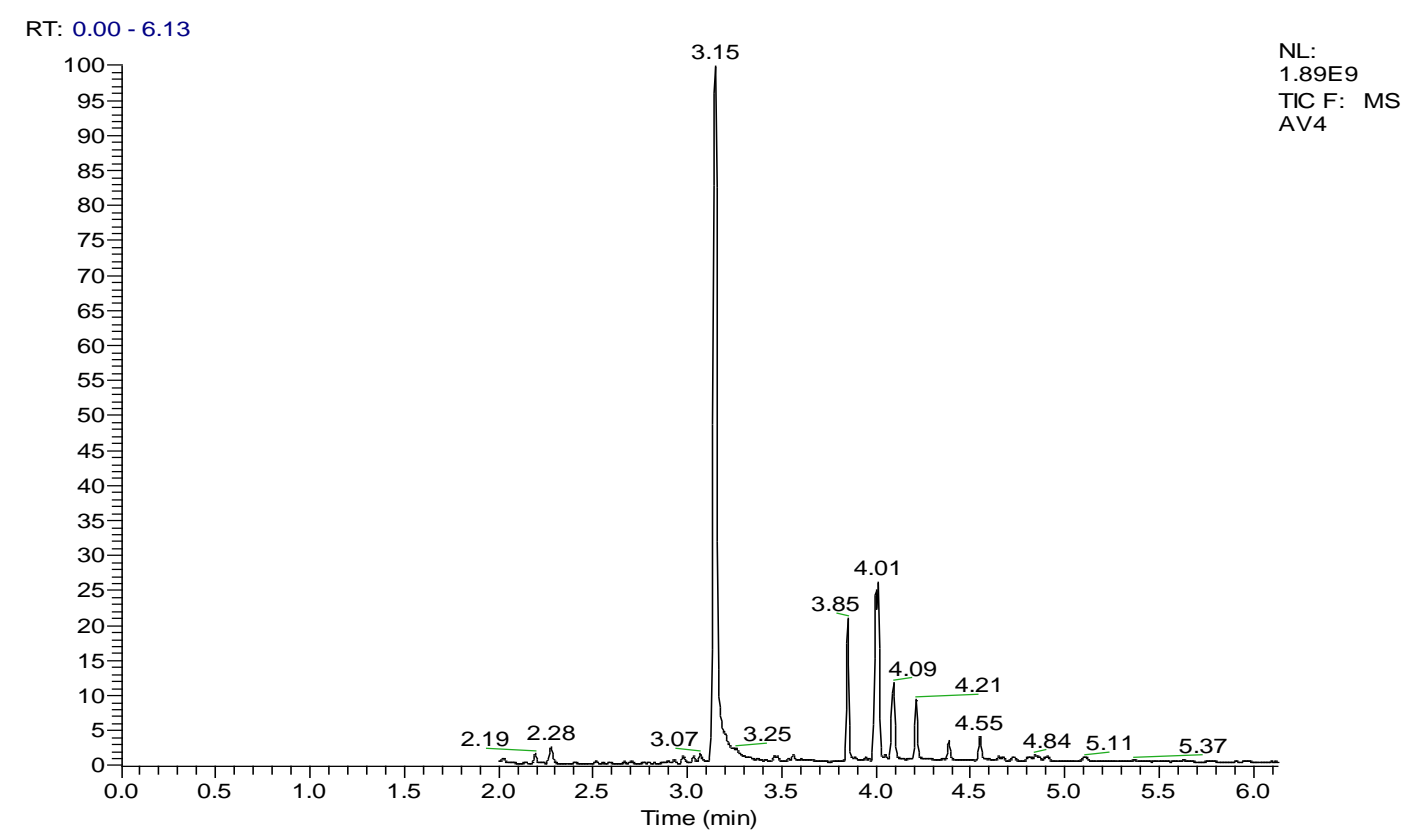

Fig. 1: Cromatograma del reactivo, solvente y producto de la termólisis de 1,2-propanodiol.

En el espectro de cromatografía figura 1, se observaron cuatro picos principales, que corresponden a los productos encontrados. El pico con tiempo de retención de 3.85 minutos, con una abundancia relativa de $23 \%$, asignado como producto 1 : $\left(P_{1-1}\right)$, el pico con tiempo de retención de 4.01 minutos, con una abundancia relativa de $28 \%$, asignado como producto 2 : $\left(\mathrm{P}_{1-2^{2}}\right)$, el pico con tiempo de retención de 4.09 minutos, con una abundancia relativa de $13 \%$, asignado como producto 3:( $\left.\mathrm{P}_{2-2^{2}}\right)$, y el pico con tiempo de retención de 4.21 minutos, con una abundancia relativa de $9 \%$, asignado como un isómero del producto 1: $\left(\mathrm{P}_{1-1^{1}}\right)$, (según el espectro de masa), son mostrados en la figura 2 y elucidados por espectrometría de masa.

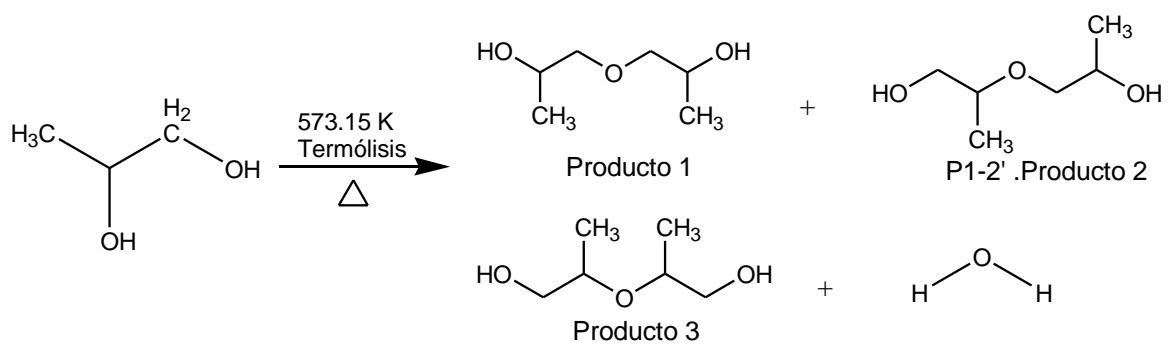

Fig. 2: Reacción general de la termólisis del 1,2-propanodiol

Para los productos elucidados por espectrometría de masa, se plantearon tres mecanismos de reacción designados como 1-1', 1-2' y 2-2' respectivamente, que difiere en la posición de los grupos $\mathrm{OH}$ que interactúan y cuyas vías de reacción se propusieron con base en cálculos computacionales, ya que no fue posible seguir la cinética de descomposición del diol experimentalmente. Además con las evidencias experimentales de los productos obtenidos claramente elucidados y con la certeza que el proceso corresponde a una dimerización, se 
plantearon mecanismos razonables para cada producto formado. En la figura 3, se muestra el espectro de masa para el producto 1 y la elucidación de los fraccionamientos de masa respectivos en la tabla 1.

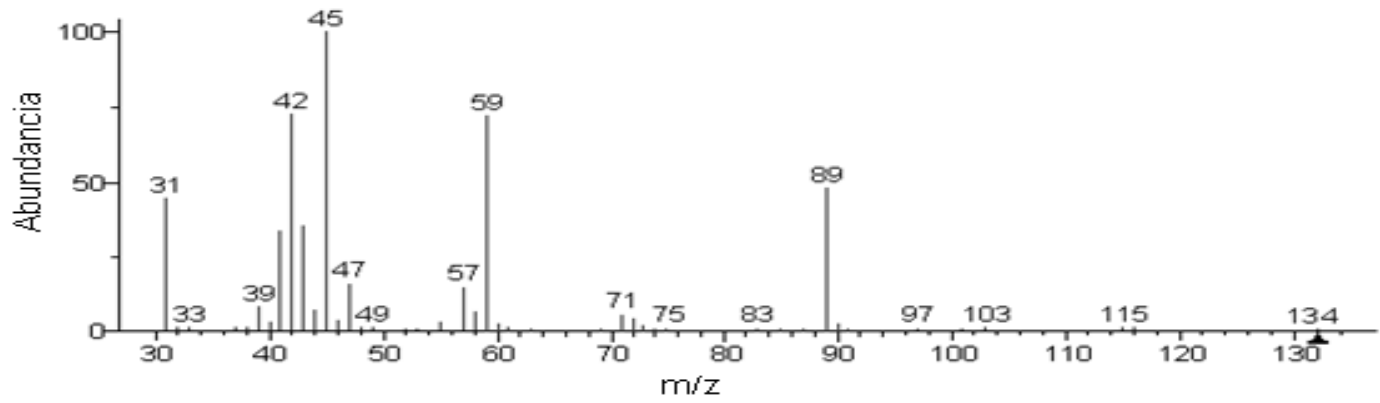

Fig. 3: Espectro de masas del producto 1

Tabla 1: Fraccionamientos de masas para el producto 1 de la termólisis de 1,2-propanodiol

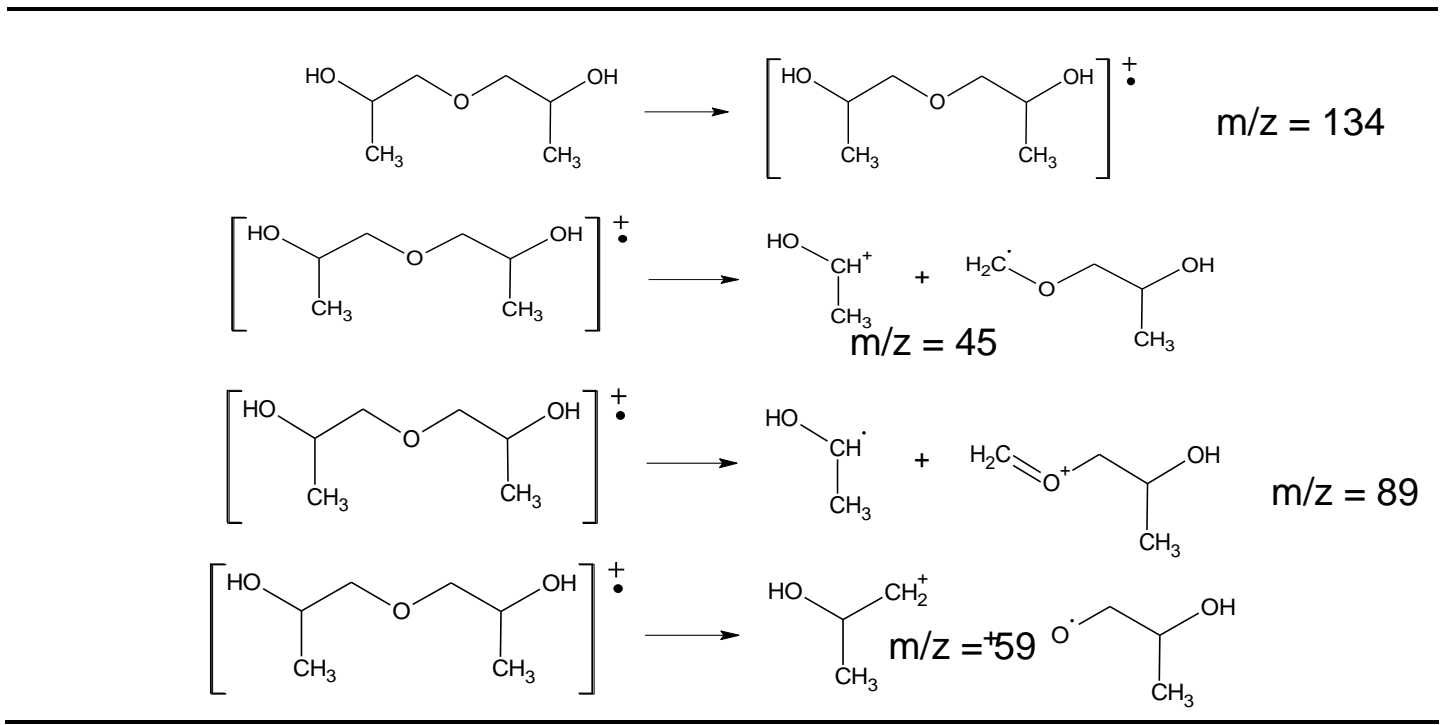

De acuerdo con el compuesto formado como producto 1 y el reactivo de partida se planteó el mecanismo de reacción, como se muestra en la figura 4, donde el átomo de oxígeno del grupo $\mathrm{OH}$ situado en el átomo de carbono 1 de una de las dos moléculas involucradas en la reacción, ataca al átomo de hidrógeno $(\mathrm{H})$ del grupo $\mathrm{OH}$ correspondiente al carbono 1' de la segunda molécula del 1,2-propanodiol, liberando una molécula de agua, en un proceso concertado de una sola etapa que pasa por un estado de transición cíclico de cuatro miembros. De ahí, que sea designado como mecanismo 1-1'.

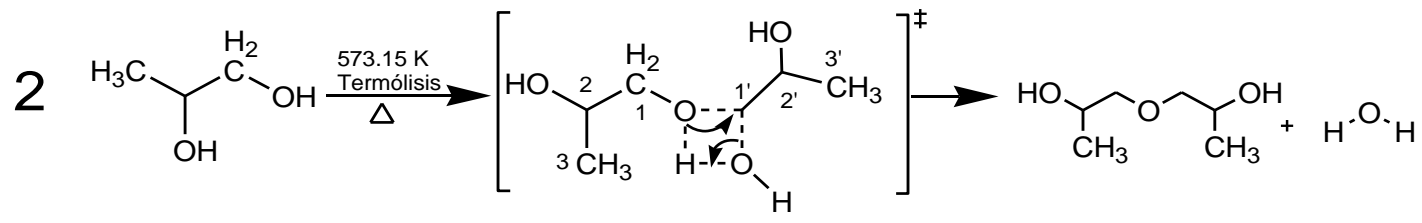

Fig. 4: Mecanismo 1-1' para el producto 1

Producto $2\left(\mathrm{P}_{1-2}\right)$ : 2-(2-hidroxipropoxi)-propanol. De acuerdo al cromatograma de la figura 1, el pico correspondiente al tiempo de retención de 4.01 minutos, corresponde al producto 2 . En la figura 5, se muestra su espectro de masa obtenido de la termólisis del 1,2-propanodiol y la elucidación de los rompimientos de masa respectivos en la tabla 2. 


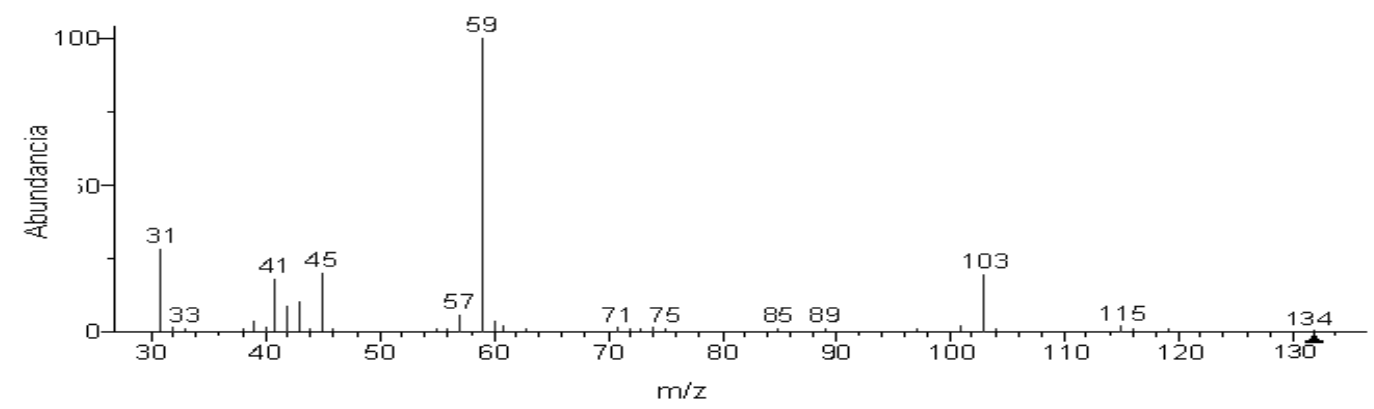

Fig. 5: Espectro de masas del producto 2

Tabla 2: Fraccionamientos de masas para el producto 2 de la termólisis de 1,2-Propanodiol

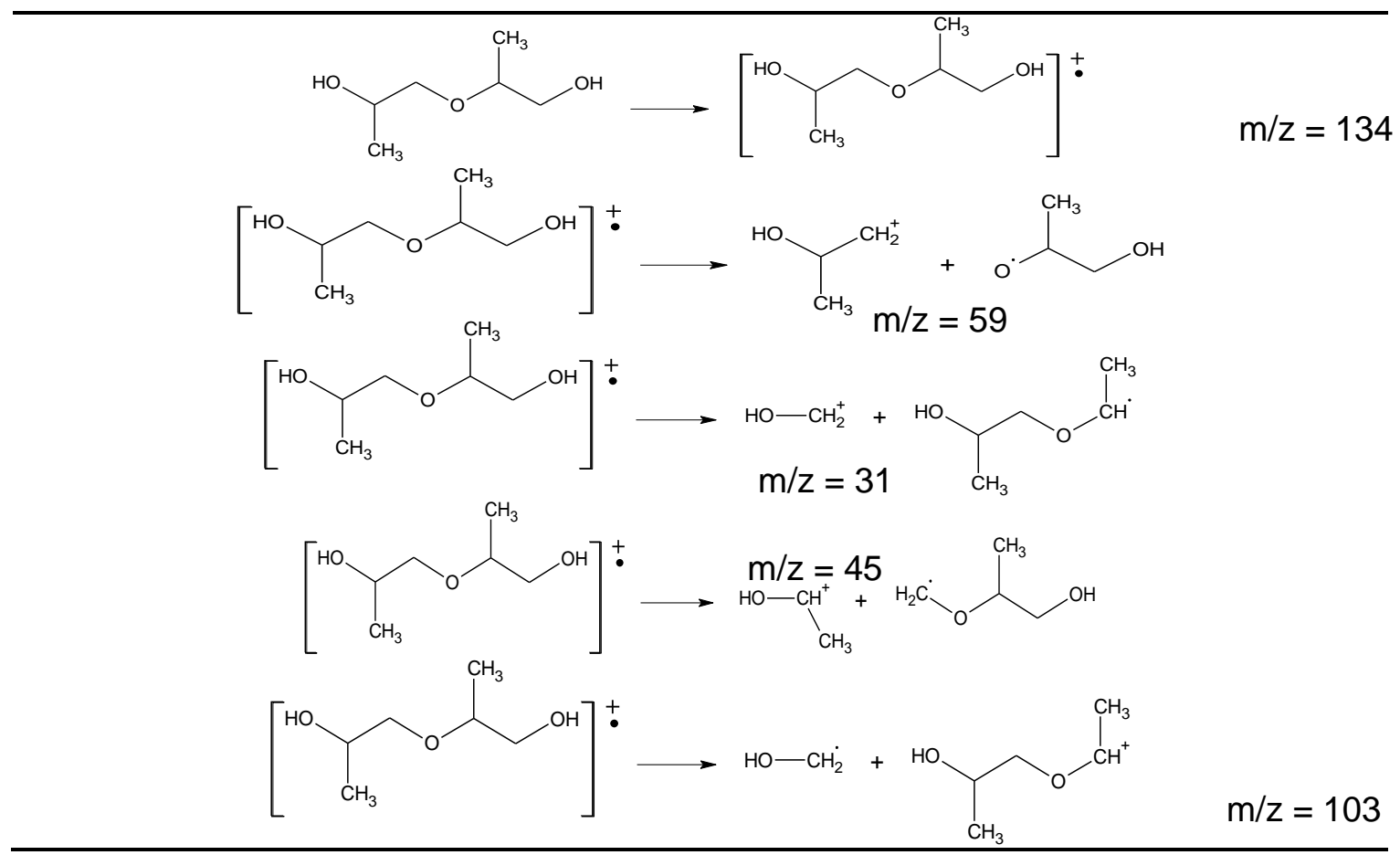

De acuerdo con el compuesto formado como producto 2 y el reactivo de partida se plantea el mecanismo de reacción, como se muestra en la figura 6 , donde el oxígeno del grupo $\mathrm{OH}$ alojado en el carbono 1 interactúa con el átomo de hidrógeno del grupo $\mathrm{OH}$ alojado en el átomo de carbono 2' de la segunda molécula y liberando agua, en un proceso concertado de una sola etapa que pasa por un estado de transición cíclico de cuatro miembros, designado como mecanismo 12 .

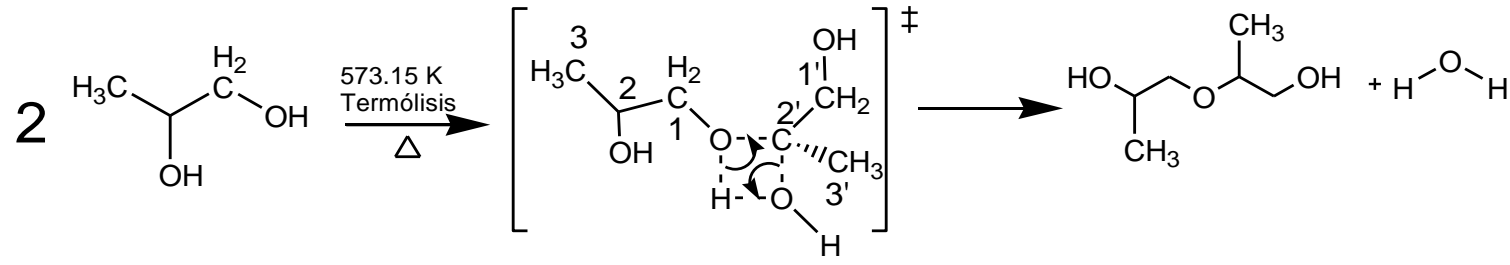

Fig. 6: Mecanismo 1-2' para el producto 2

Producto $3\left(\mathrm{P}_{2-2}\right)$ : 2-oxi-(bis)-propanol. De acuerdo con el cromatograma de la figura 1, el pico correspondiente al tiempo de retención de 4.09 minutos, corresponde al producto 3 . En la figura 7 , se muestra el espectro de masa obtenido de la termólisis del 1,2-propanodiol y la elucidación de los fraccionamientos de masa respectivos en la tabla 3. 


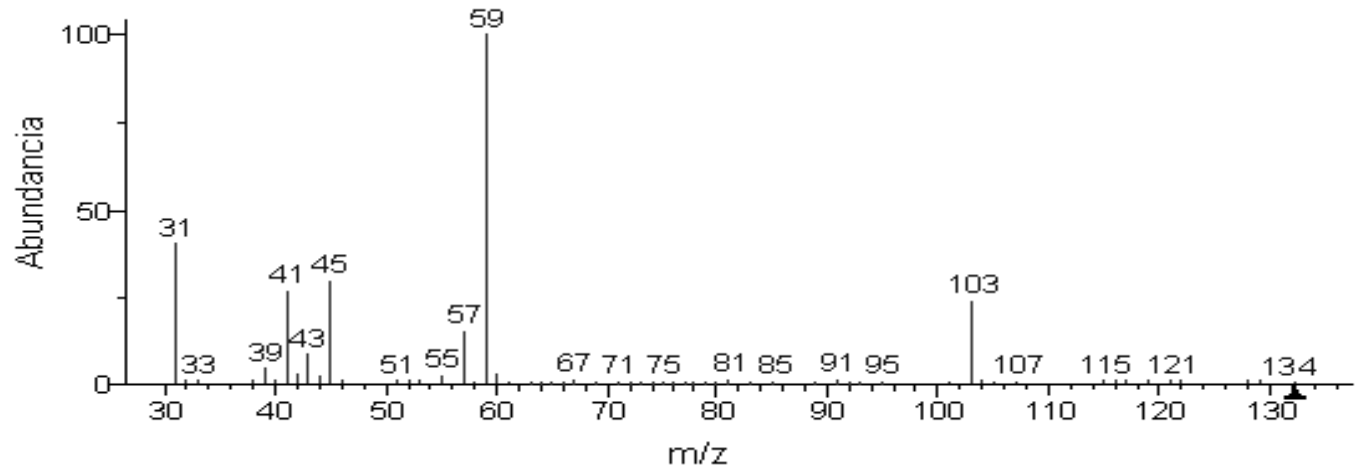

Fig. 7: Espectro de masas del producto 3

Tabla 3: Fraccionamientos de masas producto 3 de la termólisis de 1,2-propanodiol<smiles>CC(CO)O[C@H](C)O[C@H](C)CO</smiles>

De acuerdo con el compuesto formado como producto 3 y el reactivo de partida se plantea el mecanismo de reacción, como se muestra en la figura 8, el átomo de oxígeno del grupo $\mathrm{OH}$ del carbono 2 de una de las dos moléculas interactúa con el átomo de hidrógeno del grupo $\mathrm{OH}$ del carbono 2' de la segunda molécula, liberando agua, en un proceso concertado de una sola etapa que pasa por un estado de transición cíclico de cuatro miembros. Designado como mecanismo 2$2^{\prime}$.

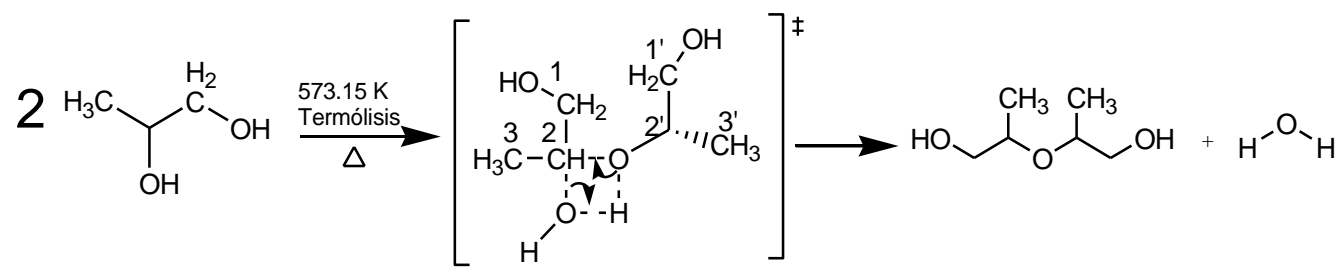

Fig. 8: Mecanismo 2-2' para el producto 3

Del estudio computacional, se obtuvieron los parámetros cinéticos y termodinámicos de la reacción de termólisis del 1,2-propanodiol, mostrados en la tabla 4, los cuales están en buen acuerdo con los resultados experimentales confirmando los datos empíricos obtenidos. La reacción de descomposición térmica del compuesto 1,2-propanodiol (1,2-PDO) generó tres productos los cuales resultaron ser isómeros que se formaron en cantidades diferentes. De los cálculos cinéticos y termodinámicos se observó que el compuesto $P_{1-2}$, tiene una constante de velocidad de formación mayor que los demás productos y una menor energía libre de activación, lo que explica por que este compuesto es más estable, confirmando los resultados experimentales 
en donde el producto $P_{1-2}$, es el más abundante con un $28 \%$ de acuerdo con los resultados de cromatografía (figura 1), por tanto se puede deducir que este compuesto se obtienen en mayor cantidad como producto y se forma más rápidamente porque presenta menor impedimento estérico, ya que los grupos sustituyentes están más alejados en el producto $\mathrm{P}_{1-2^{\prime}}$ que en los otros dos productos y además predice que la reacción tiene un control termodinámico ( figura 9.).

Tabla 4. Parámetros termodinámicos de activación y constantes de velocidad calculados al nivel B3LYP/6-31+G(d,p) para los mecanismos propuestos.

\begin{tabular}{ccccc}
\hline Mecanismo & $\Delta \mathrm{G}^{\mp}\left(\mathrm{kcal} \mathrm{mol}^{-1}\right)$ & $\Delta \mathrm{H}^{\mp}\left(\mathrm{kcal} \mathrm{mol}^{-1}\right)$ & $\Delta \mathrm{S}^{\mp}\left(\mathrm{kcal} \mathrm{mol}^{-1} \mathrm{~K}^{-1}\right)$ & ${\mathrm{k}\left(\mathrm{s}^{-1}\right)}^{-20}$ \\
\hline $1-1^{\prime}$ & 85.836 & 63.580 & -0.037 & $2.210 \times 10^{-20}$ \\
$1-2^{\prime}$ & 66.179 & 64.043 & -0.032 & $6.932 \times 10^{-13}$ \\
$2-2^{\prime}$ & 92.618 & 68.138 & -0.030 & $5.737 \times 10^{-23}$ \\
\hline
\end{tabular}

De acuerdo con los productos formados, se planteó un mecanismo general en donde el proceso es concertado de una sola etapa que transcurre por un estado de transición cíclico de cuatro miembros confirmado de acuerdo con el valor de la entropía negativa (Lafont, 2009; Ensuncho, et al., 2011). Además, los valores positivos para la entalpía de activación y energía de activación indican que la reacción de descomposición es endergónico, estable, no espontánea y con energía interna no suficiente para que la reacción química se lleve a cabo a condiciones normales (figura 9), confirmado con los valores pequeños de las constantes de velocidad de la reacción. Igualmente el perfil de energía calculado, que representan la energía del sistema para la reacción de descomposición térmica, para los compuestos estudiados, es mostrado en la figura 9.

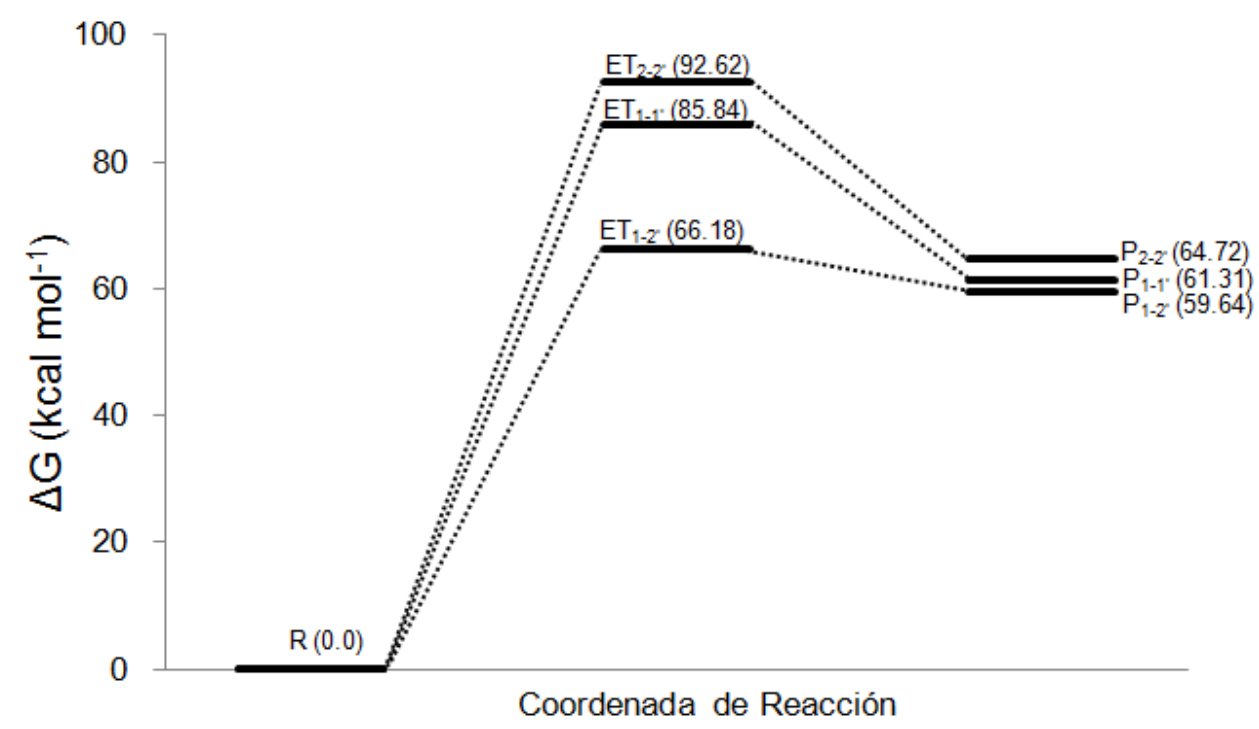

Fig.9: Perfiles de energía libre calculados al nivel B3LYP/6-31+G(d,p) a 573.15K para cada mecanismo propuesto

Asimismo, por cálculos computacionales fueron localizados los estados transición $\mathrm{ET}_{\mathrm{S}}$, por el método QST2 (Rincón y Toro-Labbé, 2007). Para cada estado de transición se obtuvo un único valor de frecuencia imaginaria, siendo $741 \mathrm{i} \mathrm{cm}{ }^{-1}$ para $E T_{1-1}$, $441.262 \mathrm{i} \mathrm{cm}^{-1}$ para $E T_{1-2}$ y $486.376 \mathrm{i}$ $\mathrm{cm}^{-1}$ para $\mathrm{ET}_{2-2}$, respectivamente. Además del criterio del valor negativo de la hessiana para la caracterización de cada estado de transición localizado se usó el método de la coordenada intrínseca de reacción (IRC). El cálculo de la vía de reacción permitió verificar que efectivamente la estructura de transición conectaba reactivos y productos.

\section{CONCLUSIONES}

Para el 1,2-propanodiol no fue posible seguir su cinética experimentalmente por el método de cromatografía gaseosa acoplada a espectrometría de masas, ya que no se observó un avance significativo en la reacción durante el transcurso del tiempo. Sin embargo, la termólisis del 1,2- 
propanodiol a 573.15K mostró como productos de reacción, los compuestos 3-oxi-(bis)-2propanol, 2-(2-hidroxipropoxi)-propanol y 2-oxi- (bis)-propanol, que resultaron ser isómeros. Aun cuando no fue posible realizar la cinética experimentalmente de la termólisis del 1,2-propanodiol, se planteó un estudio computacional para las posibles rutas mecanistica para la formación de los tres productos obtenidos, el cual resultó ser consistente con los resultados experimentales. De la termólisis 1,2-propanodiol se observó que los productos formados son compuestos dímeros, que se formaron a través de mecanismos concertados en una sola etapa de reacción, los cuales no han sido reportados como productos de termólisis.

\section{AGRADECIMIENTOS}

Al Centro de Investigaciones de la Universidad de Córdoba (CIUC), por la financiación del proyecto FCB-07-06, numeral 112020.

\section{REFERENCIAS}

Buggins, T.R., Dickinson, P.A. y G.Taylor., The effects of pharmaceutical excipients on drug disposition, Adv. Drug Deliv. Rev, 59 (15) 1482-1503 (2007).

Balci, K. y S. Akyuz., A vibrational spectroscopic investigation on benzocaine molecule, Vib spectrosc , 48(2), 215-228 (2008).

Contreras, J.G. y L.A. Gerli., Conformational preference in 4,6-dimethyl-I,3-thioxane, JCCS, 53(1), 1389-1392 (2008).

Csonka, G.I., Proper basis set for quantum mechanical studies of potential energy surfaces of carbohydrates, J. Mol. Struc-Theochem, 584(1-3), 1-4 (2002).

Chuchani, G., R.M. Dominguez, A. Rotinov, y I. Martín., The gas-phase pyrolysis of several aliphatic 1,3-diols. The kinetic and mechanism of 2,4-dimethyl-2,4-pentanediol, Inter. J. Chem. Kinet, 29 (11), 851-854 (1997).

Chopra, D, N. Tayur, G. Rowa, E. Arunan, A. Roger y K. Rudolf., Crystalline ethane-1,2-diol does not have intra-molecular hydrogen bonding: Experimental and theoretical charge density studies, J. Mol. Struc-Theochem, 964(1-3), 126-133 (2010).

Ensuncho, A., N. Milanés y J. Robles., Estudio Experimental y Computacional de la Cinética de Termólisis del 2,5-Hexanodiol, Infor. Tecnol, 22, (2011).

Eichmann, E, Alvarez, E y J.S, Brodbelt., Effects of Functional Group Interactions on the Bimolecular and Dissociation Reactions of Diols, J. Am. Soc. Mass. Spectrom, 3, 535-542 (1992).

Frisch, M. J., G. W. Trucks y H. B. Schlegel., Gaussian 03, Wallingford CT, Gaussian, Inc (2004).

Friedemann, R, A. Fengler, S. Naumann y U. Gromann., Theoretical studies on the structure of chiral 1,2-diol systems, monomers, dimers and monohydrate, J. Mol. Struc-Theochem, 357 217223 (1995).

Friedemann, R. y S. Naumann., GROMOS-MD simulations on 1,2-diol water clusters, J Mol. StrucTheochem, 398-399 405-410 (1997).

Ilieva, S., D. Cheshmedzhieva, y D. Tasheva., The origin of diastereoselectivity in the Michael addition reaction: a computational study of the interaction between $\mathrm{CH}$-acidic Schiff base and $\alpha, \beta$ unsaturated ketones, Tetrahedron, 66(27-28), 5168-5172 (2010).

Janik, M.J., R.J. Davis, y M. Neurock, M., A density functional theory study of the alkylation of isobutane with butene over phosphotungstic acid, J. Catal, 244(1), 65-77 (2006).

Kawakami, K., N. Oda, K. Miyoshi, T. Funaki y Y. Ida., Solubilization behavior of a poorly soluble drug under combined use of surfactants and cosolvents, Eur. J. Pharm. Scien, 28(1-2), 7-14 (2006). 
Levine, I.N., A. Requen, A. Bastida y J. Zuñiga., Química cuántica, Madrid, Prentice Hall (2001).

Lafont, J, M. Páez y A. Alvis., Estudio cinético de la descomposición térmica de tres derivados del pirano, Infor. Tecnol, 20(5), 39-46 (2009).

Mohajeri, A y M.J. Jenabi., Singlet and triplet potential energy surfaces of $\mathrm{C}_{3} \mathrm{H}_{2}$, J Mol. StrucTheochem, 820(1-3), 65-73 (2007).

Ochterski, J.W., Thermochemistry in Gaussian, Gaussian, Inc., 1-19 (2000).

Pawlowski, P. y G. Rokicki., Synthesis of oligocarbonate diols from ethylene carbonate and aliphatic diols catalyzed by alkali metal salts. Polymer, 45(10), 3125-3137 (2004).

Quijano, J., E. Vélez, R. Notario, C. Quijano y A. Escobar, Thermolysis of substituted Bhydroxyketones-ß-aryl. A computational study, Dyna, 73(150), 89-96 (2006).

Rogulska, M., Kultys, A. y W., Podkoscielny., Studies on thermoplastic polyurethanes based on new diphenylethane-derivative diols II. Synthesis and characterization of segmented polyurethanes from HDI and MDI, Eur. Polymer. J., 43(4), 1402-1414 (2007).

Rokicki, G. y A. Piotrowska., A new route to polyurethanes from ethylene carbonate, diamines and diols, Polymer, 43(10), 2927-2935 (2002).

Rytting, E., K.A. Lentz, X.Q. Chen, F. Qian y S. Venkatesh., Aqueous and cosolvent solubility data for drug-like organic compounds, The AAPS Journal, 7(1), 78-105 (2005).

Rincón, E. y A. Toro-Labbé, A., Reaction force and electron localization function analysis of the metal chelation process in Mg(II)-thymine complex, Chem. Phys. Lett, 438(1-3), 93-98 (2007).

Ye, L., L. Zhao, L. Zhang y F. Qi. Theoretical Studies on the Unimolecular Decomposition of Ethylene Glycol, J. Phys. Chem. A. 116, 55-63 (2012).

Zaichikov, A.M. y G. N. Levochkina, Structural and thermodynamic characteristics and intermolecular interactions in aqueous solutions of diols, J. Struct. Chem., 48 (6), 1089-1098, (2007). 